\title{
Proceso de educación intercultural en primera infancia: programa Educa a tu hijo -Cuba- y programa Hogares Comunitarios de Bienestar -HBC -Colombia-1
}

\author{
Cindy Tatiana Capera Espinel \\ Corporación Universitaria Minuto de Dios - UNIMINUTO \\ c95tatiana@gmail.com \\ Leidy Andrea Pardo Agudelo \\ Corporación Universitaria Minuto de Dios - UNIMINUTO \\ andreapardo17@hotmail.com \\ Evelyne Dahanna Torres Sandoval ${ }^{2}$ \\ Corporación Universitaria Minuto de Dios - UNIMINUTO \\ dayis_0320@hotmail.com \\ Zaily del Pilar Garcia Gutiérrez ${ }^{3}$ \\ Corporación Universitaria Minuto de Dios - UNIMINUTO \\ zaily.garcia@gmail.com
}

1 Artículo de divulgación resultado de una experiencia de internacionalización como parte del proceso de formación de pedagogos infantiles de la Corporación Universitaria Minuto de Dios - UNIMINUTO.

2 Estudiantes de Licenciatura en Pedagogía Infantil de la Corporación Universitaria Minuto de Dios UNIMINUTO.

3 Magíster en Comunicación Educativa. Líder de investigación de la Licenciatura en Pedagogía Infantil de la Corporación Universitaria Minuto de Dios - UNIMINUTO. 


\title{
Proceso de educación intercultural en primera infancia: programa Educa a tu hijo -Cuba- y programa Hogares Comunitarios de Bienestar -HBC -Colombia-
}

\section{Resumen}

En el presente artículo se pretende hacer un análisis comparativo, desde una perspectiva intercultural, de dos programas educativos no convencionales, el programa cubano Educa a tu hijo y el programa colombiano Hogares Comunitarios de Bienestar - $\mathrm{HCB}-$, dirigidos a la educación de la primera infancia. Se hace referencia a las características y estrategias en las que se evidencia el vínculo existente entre educación intercultural y la primera infancia, con el fin de fortalecer el desarrollo integral de niños y niñas. Esto surge como resultado de una experiencia de internacionalización en la que se desarrolló el curso de corta duración titulado «Didáctica de la inclusión social y la actividad física» durante dieciséis días en Cuba, ello como parte del proceso de formación de pedagogos infantiles de UNIMINUTO.

Palabras clave: primera infancia, desarrollo integral, educación intercultural, estrategias, programas educativos.

\section{Intercultural Early Childhood Education Process: Educate Your Child, -Cuba- and Community Welfare Homes Programs -HCB -Colombia-}

\begin{abstract}
This article intends to make a comparative analysis from an intercultural perspective of two non-conventional educational programs: the Cuban program Educa a tu hijo and the Colombian program Hogares Comunitarios de Bienestar -HCB-, aimed at early childhood education. Reference is made to the characteristics and strategies that demonstrate the linkage between intercultural education and early childhood in order to strengthen the integral development of children. This emerges as a result of an internationalization experience in which the short course "Didactics of social inclusion and physical activity" was developed during sixteen days in Cuba, as part of the process of training of UNIMINUTO children's educators.
\end{abstract}

Key words: early childhood, integral development, intercultural education, strategies, educational programs.

\section{Processo de educação intercultural em primeira infância: programa Educa a seu filho -Cuba- e programe Lares Comunitários de Bem-estar -HCB -Colômbia-}

\section{Resumo}

No presente artigo pretende-se fazer uma análise comparativa, desde uma perspectiva intercultural, de dois programas educativos não convencionais, o programa cubano Educa a seu filho e o programa colombiano Lares Comunitários de Bem-estar - $\mathrm{HCB}-$, dirigidos à educação da primeira infância. Faz-se referência às características e estratégias nas que se evidência o vínculo existente entre educação intercultural e a primeira infância, com o fim de fortalecer o desenvolvimento integral de meninos e meninas. Isto surge como resultado de uma experiência de internacionalização na que se desenvolveu o curso de curta duração titulado «Didática da inclusão social e a atividade física» durante dezesseis dias em Cuba, isso como parte do processo de formação de pedagogos infantis de UNIMINUTO.

Palavras-chave: primeira infância, desenvolvimento integral, educação intercultural, estratégias, programas educativos. 


\section{Introducción}

Este artículo es el resultado de una experiencia de internacionalización como parte del proceso de formación de pedagogos infantiles de la Corporación Universitaria Minuto de Dios - UNIMINUTO. La experiencia se llevó a cabo en Cuba durante dieciséis días del mes de diciembre del año 2015, en los que la Universidad de Ciencias de la Cultura Física y el Deporte, Facultad de Villa Clara —Cuba—, certifica la participación y aprobación del curso internacional de entrenamiento académico «Didáctica de la inclusión social y la actividad física». Las competencias desarrolladas a lo largo del curso giraron en torno a temas como pedagogía de la inclusión social, decolonialidad e interculturalidad, grupos poblacionales, derechos, inclusión social en la infancia, bioadaptación, teoría y práctica de los juegos, gimnasia y educación rítmica y deporte para todos; no obstante, es de mayor interés abordar los programas ya establecidos y dirigidos para la primera infancia en pro de una educación intercultural.

De acuerdo a lo anterior, en el presente artículo se pretende profundizar inicialmente sobre la educación de la primera infancia. Para esto se realiza una búsqueda bibliográfica de las características particulares del sistema educativo dirigido a una atención integral, y así, en un segundo momento, identificar y precisar los aspectos que dan paso a una educación intercultural.

Por consiguiente, se hace referencia a estrategias que se evidencian en el vínculo existente entre educación intercultural y la primera infancia, con el objetivo de fortalecer el desarrollo integral de niños y niñas. Bajo ese propósito se efectúa un análisis comparativo de programas educativos existentes en Cuba y en Colombia, a partir de una perspectiva intercultural, para dar a conocer el contenido metodológico de cada programa por medio de una breve descripción que permita distinguir sus particularidades, $y$, finalmente, de esta manera arribar a las conclusiones con base en la formación profesional como licenciadas.

\section{Educación de la primera infancia}

La educación inicial es un proceso permanente y continuo en el que, por medio de las relaciones sociales, el acompañamiento, el cuidado de un adulto y la creación de apropiados ambientes de aprendizaje, se da la posibilidad de potenciar en los niños y niñas capacidades y competencias adecuadas para su crecimiento y desarrollo integral. Durante los tres primeros años de vida el niño desarrolla la mayor parte de su cerebro, por tanto, que se dé satisfactoriamente este proceso depende en gran medida del entorno en donde el infante crece (Restrepo, 2009). 
Dentro del proceso educativo que se lleva a cabo con la primera infancia se adquieren compromisos por parte de las instituciones educativas, familia y sociedad, quienes son los agentes encargados de propiciar un proceso de desarrollo integral para los niños y niñas. Ahí, padres de familia y docentes se convierten en el modelo a seguir de acuerdo a sus acciones, lo que da las bases para la formación de personalidades y comportamientos dentro de la sociedad, donde el derecho a la educación prevalece.

Es importante resaltar que la educación inicial no busca como último fin la preparación para el nivel primaria, sino que, antes bien, ofrece retos al propiciar experiencias que impulsen su desarrollo desde la implementación del juego, el arte, la literatura y la exploración del medio, los cuales son considerados como pilares de la educación inicial, de acuerdo a la Secretaría de Integración Social del Distrito quien los establece en los Lineamientos curriculares para la primera infancia (2008), o actividades rectoras, en palabras del Ministerio de Educación Nacional.

En este sentido, las acciones educativas de este nivel deben estar dirigidas a potenciar el desarrollo cognitivo, físico, emocional, lingüístico, motor, social, moral y sexual, con el propósito de desarrollar todas sus capacidades. Para ello es fundamental el aporte de diferentes ciencias como la psicología, la filosofía, la pedagogía y la neurología, entre otras; las cuales, gracias a sus diferentes estudios, han hecho un aporte significativo a la comprensión de las bases de las acciones pedagógicas en la educación inicial.

La Constitución Política de Colombia (1991) en el artículo 44 determina la educación como un derecho fundamental de los niños y las niñas, a su vez, el Código de la Infancia y la Adolescencia determina que:

[...] la primera infancia es la etapa del ciclo vital en la que se establecen las bases para el desarrollo cognitivo, emocional y social del ser humano. Comprende la franja poblacional que va de los cero (0) a los seis (6) años. Son derechos impostergables de la primera infancia: la atención en salud y nutrición, el esquema completo de vacunación, la protección contra los peligros físicos y la educación inicial. (Ley 1098 de 2006, art. 29).

En esta etapa, la intención de la educación inicial es dinamizar el significado que tiene para el ser humano asistir a un centro educativo en sus primeros seis años de vida por medio de acciones dirigidas a la atención de los niños y niñas, con el objetivo de promover su desarrollo integral a partir de las características y el contexto donde se encuentran y favorecer interacciones con el entorno.

En Colombia existe un gran reto para garantizar el cumplimiento del derecho a la educación inicial y al desarrollo integral de la primera infancia, así lo determina 
Proceso de educación intercultural en primera infancia: programa Educa a tu hijo -Cuba- y programa Hogares Comunitarios de Bienestar -HCB -Colombia| Cindy Tatiana Capera Espinel | Leidy Andrea Pardo Agudelo | Evelyne Dahanna Torres Sandoval | Zaily del Pilar García Gutiérrez |

el Plan Decenal de Educación (2006-2015); por ende, el Ministerio de Educación Nacional implementa una política educativa que busca garantizar la atención integral a niños y niñas menores de cinco años. Esta estrategia se divide en cinco líneas de acción dentro de las cuales se encuentra el acceso de los niños menores de cinco años a una atención educativa, la construcción de centros de atención integral para la primera infancia, la formación de agentes educativos responsables del proceso que se lleva a cabo con los niños, la implementación de una política de educación inicial y, por último, el sistema de acreditación de calidad en la prestación de este servicio.

Una educación de buena calidad, una adecuada atención y cuidado son factores importantes en los procesos cognitivos, físicos y sociales de los niños, pues contribuyen al desarrollo de sus capacidades y a la adquisición de competencias para que logren un pleno progreso como seres humanos y sujetos de derechos. Así, «toda la serie de actividades educativas tienen lugar en distintos marcos y están encaminadas a satisfacer las necesidades básicas del aprendizaje» (Unesco, 1999).

En el artículo «Una mirada a la caracterización de la primera infancia: contextos y métodos», escrito por Pulido y Giraldo (2011), se establecen los programas dirigidos a la primera infancia por parte de las entidades educativas como el principal método para alcanzar un desarrollo integral en la niñez. Los programas dan a conocer una amplia gama de competencias y habilidades a lograr por los infantes que facilitan el desarrollo motor, social, cognitivo y emocional. Asimismo, se evidencia un diagnóstico situacional del estado actual en el que se encuentra la primera infancia con respecto a la integración de los programas educativos diseñados y dirigidos especialmente a esta población con el fin de lograr un desarrollo integral, puesto que el ingreso del niño y la niña a la escuela se constituye en un factor determinante para el desarrollo de habilidades y potencialidades (Selowsky, 1976).

El Ministerio de Educación Nacional (2014) presenta en su plan Modalidades y condiciones de calidad para la educación inicial la delegación de responsabilidades y funciones para los entes educativos y los ambientes de aprendizaje, en torno a una educación de calidad para la primera infancia, en el que se definen las modalidades de atención para obtener y lograr un enlace al reorganizar los servicios y unificar criterios en los diferentes sectores de educación infantil y, de ese modo, alcanzar un desarrollo integral. Es preciso reconocer que el escenario eminente en el cual se inicia la educación y formación de los niños y las niñas es la familia, pues este es el primer agente socializador y educador. Aun así, es necesario intervenir para dirigir y generar condiciones que promuevan el desarrollo integral; estos escenarios se presentan a través de dos modalidades: familiar e institucional o centros de desarrollo infantil.

Las dos modalidades están orientadas a garantizar el derecho a una educación integral y de calidad en el nivel de inicial. 


\section{Modalidad institucional: Centros de Desarrollo Infantil - $\mathrm{CDI}-$}

Estos Centros de Desarrollo Infantil se conocen como instituciones dirigidas a promover un desarrollo integral a través de la educación inicial. Cuentan con profesionales en los temas relacionados con los diferentes componentes de la atención integral y son responsables de gestionar y hacer cumplir los derechos de los niños y las niñas.

\section{Modalidad: educación inicial familiar}

Esta modalidad es considerada no convencional, pero posibilita el desarrollo integral del niño en un ambiente familiar y comunitario. Establece como punto de entrada el acompañamiento y fortalecimiento de los miembros de la familia y cuidadores para potenciar el desarrollo de los niños y niñas. El lugar protagónico de la familia en el cuidado, crianza, educación y desarrollo de los niños y las niñas, desde el nacimiento hasta antes de cumplir los seis años, la convierte en el primer y más inmediato escenario de corresponsabilidad. Para tal fin, el Estado, responsable del cumplimiento de los derechos de la primera infancia, está en la obligación de garantizar esta atención y proveer el apoyo necesario a la familia para llevar a cabalidad dicha corresponsabilidad en la atención integral.

La primera infancia se ubica dentro de diversos contextos en los que se da inicio a su proceso de formación integral, por ello, la interculturalidad se presenta como un factor decisivo en el reconocimiento a la diversidad existente dentro del aula y el entorno del que hacen parte niños y niñas, ya que, a partir de este reconocimiento, se da paso a la construcción de relaciones e identidades para el fortalecimiento de una sociedad equitativa e intercultural.

\section{Educación intercultural e interculturalidad}

En este segundo momento de la revisión, se pretende ubicar el tema de educación intercultural a partir de las definiciones e ideas que exponen algunos autores en sus respectivas publicaciones donde estudian, analizan y construyen su discurso con base en una mirada reflexiva sobre el concepto de interculturalidad a nivel social y educativo.

En América Latina el termino interculturalidad se constituye a partir de la necesidad de reconocer varios aspectos y características sociales propias de cada cultura y, por ende, de cada contexto, es decir, para poder hablar de interculturalidad es imprescindible apreciar la diversidad existente en cada una de las culturas inmersas en los contextos sociales, es allí donde se establece entre culturas un intercambio de saberes, ideas, costumbres, creencias y hábitos (Walsh, 2004). 
Proceso de educación intercultural en primera infancia: programa Educa a tu hijo -Cuba- y programa Hogares Comunitarios de Bienestar -HCB -Colombia| Cindy Tatiana Capera Espinel | Leidy Andrea Pardo Agudelo | Evelyne Dahanna Torres Sandoval | Zaily del Pilar García Gutiérrez |

Para que se lleve a cabo el proceso de interacción cultural se requiere aceptar las diferencias mediante la referencia a las cualidades y caracteres propios que definen la individualidad de cada persona y grupo que pertenece al mismo entorno, dado que es allí donde se inicia la construcción de la identidad por medio de la consolidación de relaciones fundamentadas en el respeto y la tolerancia como bases de una convivencia social estable y enriquecedora.

Según González (2011) en su obra titulada Educación intercultural en aulas de educación infantil, la interculturalidad abarca los principios de convivencia ciudadana, dados a través de la interacción que se da entre las culturas a partir de la necesidad de convivir y desarrollarse merced a la construcción de relaciones entre diferentes grupos culturales, reconociendo así el pluralismo cultural.

Uno de los elementos clave a la hora de hablar de interculturalidad es la transformación de un ambiente de desigualdad y exclusión por un ambiente equitativo e igualitario a nivel social, sin embargo, los sistemas políticos y sociales influyen, según ciertos intereses, en el discurso de interculturalidad. Walsh (2004) afirma:

Se limita la interculturalidad al contacto y a la relación — muchas veces a nivel individual-, encubriendo o dejando de lado las estructuras de la sociedad sociales, políticas, económicas y también epistémicas- que posicionan la diferencia cultural en términos de superioridad e inferioridad. (p. 3).

Se instaura una estratificación social como resultado del impacto de la colonización en la configuración de las sociedades americanas, allí sobresalen las diferencias e inequidades en cuanto a posibilidades u oportunidades socioeconómicas. En tales contextos, la sociedad vale por lo que posee a nivel material, y no por lo que posee como esencia, siendo esta esencia su cultura, la cual debe ser defendida y valorada desde la construcción de estrategias y aportes que alimenten las ideas, saberes o experiencias de cada una, y hacer patente, de esa manera, el enriquecimiento, evolución y renovación social.

En cuanto estrategia de progreso cultural y social, Walsh en su escrito Interculturalidad crítica y pedagogía de-colonial: apuestas (des)de el in-surgir, reexistir y re-vivir. Manifiesta como posibilidad de reflexión, la construcción de una interculturalidad crítica como medio para repensar y rediseñar los esquemas políticos y sociales que se han convirtiendo en obstáculos para desarrollar ambientes que disminuyan el nivel de discriminación. En tal interculturalidad se ha de reconocer a la cultura como medio de expresión, de identidad, de particularidad; así las cosas, la diferencia no se considera un factor negativo, sino que se aprovecha para unir, crear e idealizar una sociedad diversa, inclusiva, integradora e intercultural. 
Se requiere poder abrir la mente y comprender la interculturalidad como una forma de comunicación e interacción constante entre diferentes grupos culturales, en la que no sólo se reconoce y respeta la identidad de las demás personas, sino que además, a partir de las diferencias existentes, se diseña y estructura la realidad social (Lazos, 2013).

La educación entra allí como herramienta que le apuesta a observar, reflexionar y acoger todos los aspectos clave de la interculturalidad, que puede evidenciarse en la población ubicada en el contexto educativo, a partir de la necesidad de propiciar ambientes inclusivos en los que se construya igualdad y equidad de oportunidades. La interculturalidad radica en la necesidad de visibilizar, enfrentar y transformar las estructuras e instituciones que diferencialmente posicionan grupos, prácticas y pensamientos dentro de un orden y lógica que, al mismo tiempo y todavía, es racial, moderno-occidental y colonial: «Un trabajo que procura desafiar y derribar las estructuras sociales, políticas y epistémicas de la colonialidad, y alentar nuevos procesos, prácticas y estrategias de intervención para críticamente leer el mundo» (Walsh, 2004, p. 15).

Dentro del ámbito educativo se encuentra una gran diversidad cultural, y se inicia la construcción de valores e identidades de cada sujeto gracias a la intervención de estrategias que permiten ir reconociendo la diversidad como posibilidad de aprendizaje y desarrollo humano integral.

\section{Educación intercultural en la primera infancia: estrategias para el desarrollo integral}

Como paso al reconocimiento de la diversidad dentro del ámbito educativo, surge el concepto de interculturalidad a mediados del siglo xx. En él se da el reconocimiento de los derechos universales a comunidades étnicas, sociales y culturales —entre ellos, el derecho a la educación-; se comprende así que no existe una sola cultura que prevalezca ante las demás, por el contrario, se habla de un respeto e igualdad en los sistemas sociales en cuanto a cultura, religión, descendencia y demás.

Cuando se habla de primera infancia en Colombia se hace referencia a los niños y niñas menores de cinco años, los cuales nacen y crecen en un contexto cultural específico, forman parte de un tiempo y ocupan un lugar en la sociedad que les brinda una identidad cultural y particular. Tener en cuenta el ambiente sociocultural donde el niño y la niña se desenvuelven es uno de los principales requisitos para hablar de un proceso enseñanza-aprendizaje, el cual no debe recaer sólo en los educadores, sino en todo aquel que esté en la capacidad de compartir un conocimiento.

La educación intercultural asume un reto frente a la formación y desarrollo integral en la primera infancia, es decir, se habla de una educación intercultural que 
Proceso de educación intercultural en primera infancia: programa Educa a tu hijo -Cuba- y programa Hogares Comunitarios de Bienestar -HCB -Colombia| Cindy Tatiana Capera Espinel | Leidy Andrea Pardo Agudelo | Evelyne Dahanna Torres Sandoval | Zaily del Pilar García Gutiérrez |

reconozca, acepte e involucre la diversidad cultural y, a partir de ello, transforme y dé las bases para la construcción de identidad, logrando que niños y niñas reconozcan la diversidad como ente existente en el aula y en su entorno (Porlan, 2006). Por ello, la educación, más específicamente la educación de la primera infancia, no puede estar ajena a este concepto, antes bien, debe acogerlo dentro de su currículo educativo.

Los sistemas educativos están Ilamados a construir currículos que promuevan una educación intercultural. Las comunidades educativas deben realizar sus proyectos pedagógicos con suficientes bases teóricas que proporcionen una apropiada comprensión del tema y de lo que se pretende implementar para el mejoramiento de las relaciones personales, todo eso teniendo en cuenta que la adecuada integración del multiculturalismo fortalece el sentido de pertenencia e identidad en los niños y niñas, ya que estos se encuentran en una etapa de desarrollo flexible en la que adquieren con mayor facilidad conceptos y aptitudes para un desarrollo personal y social.

Por consiguiente, es menester resaltar la importancia de la creación de currículos, planes educativos, proyectos pedagógicos, programas para la primera infancia y demás, que muestren un avance hacia la interculturalidad, así como lo postula Nélida Céspedes en su obra Educación popular, interculturalidad y buen vivir, quien referencia a Freire (2010) en su discurso sobre la Educación Popular —EP- con respecto a las propuestas interculturales en las que la idea es construir currículos desde nosotros mismos y con apertura a otros ámbitos culturales.

Para favorecer un proceso de educación intercultural para la primera infancia, Peralta (2014), vicepresidente de la Organización Mundial para la Educación Preescolar - OMEP-, plantea cinco criterios a tener en cuenta en la creación de un plan curricular en instituciones dirigidas a la educación inicial. En primera instancia, es primordial contar con agentes educativos que permitan desarrollar proyectos pedagógicos con base en fundamentos sociales, antropológicos y culturales, con la habilidad de promover espacios de interculturalidad.

En segunda instancia, la familia adquiere una participación importante dentro de este proceso educativo, pues la mayoría de veces su participación va más dirigida a la colaboración en cuanto recursos, alimentos o creación de centros educativos; sin embargo, es necesario considerar a la familia como educadores irremplazables, poseedores de saberes con un gran significado para los niños, más allá del derecho de provenir de sus padres.

En tercera instancia, la revisión y el análisis del currículo, tanto nulo como oculto, es prioritaria para realizar un diagnóstico situacional de los niños, las niñas y las familias, y, a su vez, lograr la calidad en la propuesta que se quiere aplicar con la implantación de prácticas educativas multiculturales. 
En cuarto lugar, el desarrollo curricular debe implementar objetivos y prácticas educativas dirigidas a la creación de un contexto multicultural, para de esa forma, como quinto criterio, seleccionar los ejes culturales significativos que permiten la apertura de otros ámbitos culturales que dan paso al trabajo cooperativo con la población que se trabaja.

Dentro del ámbito educativo es patente el acercamiento a la diversidad cultural; en tal ámbito asisten niños y niñas de diferentes contextos sociales que inician un proceso de integración con compañeros y docentes. Muñoz (2013) afirma: «Uno de los desafíos de la educación actual para la primera infancia es la formación docente desde y para la diversidad» (p. 126). Es allí donde el rol del docente empieza a tomar más compromiso y valor, ya que este debe comprender y reconocer la diversidad del aula para diseñar e implementar estrategias que fomenten un ambiente equitativo e igualitario, siendo esto la base para un desarrollo integral.

Según la Unesco, en sus Directrices sobre la educación intercultural - SF- se plantean tres principios fundamentales, a saber:

- La educación intercultural respeta la identidad cultural del educando impartiendo una educación de calidad que se adecue y adapte a su cultura.

- La educación intercultural enseña a cada educando los conocimientos, las actitudes y las competencias culturales necesarias para que pueda participar plena y activamente en la sociedad.

- La educación intercultural enseña a todos los educandos los conocimientos, las actitudes y las competencias culturales que les permiten contribuir al respeto, el entendimiento y la solidaridad entre individuos, grupos étnicos, sociales, culturales, religiosos y naciones.

Asimismo, los principios anteriormente mencionados pretenden educar al docente, en este caso, con el fin de abordar estrategias que lleven a un proceso de igualdad en todas las formas de educación para todos los grupos culturales.

Como estrategia prevalece la comunicación y uso del lenguaje en cuanto medio de interacción en el que, a partir de la expresión de ideas, pensamientos y experiencias, se va fortaleciendo la construcción de identidad de niños y niñas, lo que logra la formación de competencias y habilidades que permiten:

Desarrollar la capacidad de entender la propia cultura desde la acción y la reflexión, o, en términos más clásicos, desde la teoría y desde la práctica, reconociendo cómo aquella influye determinantemente en la forma en que nos vemos a nosotros mismos y a los demás. (Porlan, 2006, p. 877). 
Proceso de educación intercultural en primera infancia: programa Educa a tu hijo -Cuba- y programa Hogares Comunitarios de Bienestar -HCB -Colombia| Cindy Tatiana Capera Espinel | Leidy Andrea Pardo Agudelo | Evelyne Dahanna Torres Sandoval | Zaily del Pilar García Gutiérrez |

Por lo tanto, debido al lenguaje, los niños y niñas pueden relacionarse en determinados contextos y situaciones para así reconocer a los demás y a sí mismos como personas únicas, que actúan, piensan, sienten y viven bajo diversas ideas y creencias que hacen posible hablar de diversidad social. Agudelo y Estupiñán (2009) sostienen que: «Los sujetos al relacionarse permiten los encuentros interculturales, tejiendo la trama de sus realizaciones en escenarios institucionales. Este es un encuentro de seres humanos, de seres creativos, de seres diversos que pueden transformar situaciones, escenarios y saberes» (p. 85). Ello da paso a la construcción de relaciones interpersonales entre niños, niñas y docentes para favorecer el intercambio de experiencias y saberes que aporten a la formación de su personalidad.

Por otro lado, Muñoz (2013) plantea que «a través del lenguaje las niñas y niños potencian habilidades cognitivas y sociales que les permiten construir sus propias identidades, en cuanto éstas se desarrollan en las relaciones dialógicas que se establecen en la escuela» (p. 129). Como parte de un desarrollo integral se busca que niños y niñas puedan fortalecer y construir las bases de su aprendizaje por medio del cuidado y apoyo para potenciar capacidades cognitivas y sociales que les permitan desenvolverse en la sociedad presente y futura.

Cabe resaltar que la construcción de las bases de aprendizaje se ven influenciadas por entes sociales como la familia y la comunidad, quienes cumplen un papel fundamental y son partícipes de estrategias diseñadas para dar inicio a un proceso de educación intercultural desde el nacimiento y sus próximos años de vida.

\section{Programas educativos, una estrategia para la educación intercultural}

En vista de la importancia e impacto de la educación intercultural en la primera infancia, merced al acercamiento de cada concepto, se realizó la búsqueda de dos programas, uno de Colombia y otro de Cuba, como consecuencia del proceso de internacionalización que se realizó en dicho país. Estos programas son objeto de análisis y comparación; así, en este acápite se exponen las características, particularidades y aportes propios de cada programa y, a partir de ello, se establece una comparación en la que se destacan diferencias y semejanzas referentes a la propuesta planteada para la atención integral de la primera infancia desde una perspectiva intercultural.

En primera instancia, se estudiará el programa Educa a tu hijo, el cual es de procedencia cubana, un país que se caracteriza por ser líder en educación y atención a la primera infancia debido a la gran cobertura que realiza en América Latina y El Caribe con obras dirigidas desde la gestación hasta el ingreso a la educación primaria (Ferreiro et al, 2002). 
Antes de dar a conocer el contenido del programa Educa a tu hijo, es prudente hacer un breve acercamiento a los antecedentes relacionados con la primera infancia para, de ese modo, comprender la necesidad de diseñar y aplicar una estrategia que atienda a dicha población en Cuba; frente a ello, la docente Ana María Siverio Gómez (2011) nombra algunos antecedentes relevantes sobre primera infancia, políticas públicas, y algunos acontecimientos que aportaron a la construcción y creación del programa Educa a tu hijo. Para iniciar, en la República de Cuba surge un cambio social a partir del año 1959, en el que se crea la Ley 64 dentro de la constitución política del gobierno, la cual centra su atención en la infancia y la juventud para preservar sus derechos y su pleno desarrollo integral.

Adicionalmente, el Estado cubano formula políticas económicas, sociales y culturales con el fin de alcanzar equidad y justicia en la población; partiendo de ello, la educación y la salud se establecen como sectores prioritarios. El Sistema Nacional de Salud garantiza la atención a toda la población y en especial a la primera infancia por medio de programas y proyectos como el Programa Nacional de Vacunación, atención materno infantil, neuroestimulación temprana, programas de salud escolar, entre otros.

El Sistema Nacional de Educación adquiere una dimensión humanista bajo el propósito de que la educación sea un derecho para todos. En 1961 se realiza una campaña de alfabetización que se extiende rápidamente a cada rincón del país, la cual marca un hito en el cuidado y la atención a los niños y niñas en sus primeros seis años de vida, además, se crea el instituto de infancia y se inicia la promulgación de leyes y decretos en pro del desarrollo integral de los niños y niñas, los cuales dan paso a la realización de programas educativos dirigidos a esta población.

A inicios de la década de 1980 se planteó la necesidad de ofrecer el programa en favor de una atención integral a los niños y niñas que viven en zonas rurales, apartadas o dispersas, y que, por tal motivo, no pueden acceder a un plantel educativo, a una preparación para el aprendizaje escolar.

El programa social de atención educativa Educa a tu hijo inicia con una investigación que va desde el año 1982 hasta 1992, en la cual se concibe a la familia como el agente protagónico encargado de favorecer y promover el desarrollo integral del niño desde antes de su nacimiento hasta el ingreso a la escuela primaria. El estudio se inició con un grupo integrado por pedagogos, psicólogos, pediatras, nutricionistas, especialistas en crecimiento y desarrollo, en cultura física, higienistas y sociólogos. Estos resultados quedaron plasmados en una colección de nueve folletos en los que se trabajan motricidad, habilidades cognitivas, comunicación y lenguaje, relaciones socioafectivas y formación de hábitos.

La atención a la primera infancia dentro de Cuba se brinda institucionalmente en círculos infantiles y aulas de preescolar-conocidos en Colombia como jardines 
infantiles--; de otro lado, se ofrece una vía no institucional en comunidades y hogares por medio del programa Educa a tu hijo. De esa manera lo plantea el Ministerio de Educación y el Sistema cubano de educación inicial.

En 1992, Educa a tu hijo fue aprobado por el Ministerio de Educación. Posteriormente, en el año 1993, fue considerado por la Academia de Ciencias de Cuba como «Resultado Científico Destacado» y fue premiado. La cobertura que realizó el programa (2003) fue del 70.9 \% de la población infantil, de zonas rurales y urbanas, comprendida entre los cero y seis años. Actualmente, la población total de niños y niñas cubanos entre cero a seis años es de 721 184, de los cuales 479972 están vinculados al programa Educa a tu hijo. De esta manera, uno de los aspectos clave dentro del programa es contemplar el derecho de igualdad de acceso que tienen los niños y niñas al conocimiento y la cultura entre pueblos y comunidades, lo cual se puede concebir como muestra de la educación intercultural presente en el proceso de formación en la primera infancia (Unicef, 2003).

El programa tiene sus fundamentos en los principios de la pedagogía marxista y martiana: «Necesitamos otra educación para otra sociedad y otra sociedad para otra educación» (Marx, 2009). Por eso reconoce al adulto como el mediador fundamental que, en un proceso de interacción permanente con los niños y las niñas, promueve una experiencia histórico-social.

Durante el proceso educativo y de preparación, el adulto desempeña un rol de guía que orienta y organiza las acciones a partir del conocimiento de cada etapa particular del desarrollo de niños y niñas en pro de que este se logre. Allí serán significativas las experiencias y relaciones, las cuales impactarán a lo largo de dicho proceso, ya que permiten despertar el interés de niños y niñas por conocer, explorar y comprender su entorno y lo que habita en él.

Por ende, se reconoce a la familia como un pilar que influye en la educación infantil, puesto que, desde los primeros años de vida, ésta se encarga de dar una fuerte base emocional en el desarrollo social, físico e intelectual del niño y la niña. Por esta razón, a través del programa se busca que las familias y la comunidad en general adquieran el conocimiento necesario para, adecuadamente, ser partícipes en el proceso educativo de sus hijos mediante acciones educativas que den cuenta que en Cuba «la educación es tarea de todos» (Ferreiro et al, 2002).

El programa Educa a tu hijo (2011) reconoce tres elementos básicos para llevar a cabo un proceso completo de desarrollo en los niños y niñas a los que va dirigido. En el inicio se encuentra la familia como portadora de la experiencia social para cada niño; por consiguiente, ésta asume el papel protagónico, pues a partir de sus saberes y experiencias se capacita para lograr que los niños y las niñas alcancen cada una de las competencias relacionadas con su desarrollo integral. En 
segundo lugar, se encuentra la comunidad como el escenario acorde al proceso de enseñanza-aprendizaje, el cual tiene en cuenta el entorno y el contexto en el que el niño se desenvuelve. Finalmente, está el enfoque intersectorial, que hace referencia a las organizaciones que promueven e incentivan con responsabilidad el trabajo por un mismo objetivo: el bienestar y el desarrollo de los pequeños habitantes de la comunidad mediante, por ejemplo, organismos de salud, educación, cultura, deportes, protección, etc.

Se reconoce el programa como un complemento para brindar, fortalecer y propiciar una atención integral a la primera infancia. Se hace partícipe a la familia y comunidad, quienes se constituyen en entes influyentes que contribuyen en la formación y en el proceso educativo de niños y niñas con base en el desarrollo y cuidado, en cada contexto, por medio de la estimulación temprana.

El Fondo de las Naciones Unidas para la Infancia —Unicef-, en conjunto con la Organización de Estados Iberoamericanos — OEl— (2011), expone el programa social de atención educativa Educa a tu hijo de una manera integral, resaltando la importancia del desarrollo y bienestar de los niños y niñas, desde su nacimiento hasta los seis años de vida, y el papel fundamental que cumple la familia como agente primordial en el proceso de educación.

El desarrollo del niño y la niña debe ser armónico e integral, y este depende de las estrategias que fueron implementadas en la educación desde la primera infancia. Ferreiro et al. (2002) argumentan que:

El fin del sistema de educación inicial y preescolar es lograr el máximo desarrollo posible para cada niño y niña, comprendiendo este como un derecho que abarca la atención a la salud, la nutrición, el desarrollo intelectual, socioafectivo, motriz y físico, todo lo cual contribuye a una mejor preparación para el aprendizaje escolar. (p. 21).

A partir de lo anterior, dentro del programa se establecen metodologías que dan una forma coherente y ordenada a la estructura, antes, durante y después de la implementación de la misma. La creación de un grupo coordinador es la primera acción a realizar, y trata de convocar a todos los organismos, instituciones y asociaciones gubernamentales y no gubernamentales con el propósito de analizar la importancia y la necesidad de la atención integral. De otra parte, se hace necesario diseñar un plan de acción, tarea encargada por el grupo coordinador, en el que se planifica cuidadosamente el inicio exitoso de la aplicación del programa, pone en funcionamiento un proceso de sensibilización, diagnóstico, divulgación, reconocimiento, capacitación y organización de las modalidades de atención, seguimiento y evaluación. Cada proceso que se lleva a cabo se realiza bajo el más adecuado y minucioso manejo por parte de todo el equipo de trabajo, lo que arroja excelentes resultados. 
Proceso de educación intercultural en primera infancia: programa Educa a tu hijo -Cuba- y programa Hogares Comunitarios de Bienestar -HCB -Colombia| Cindy Tatiana Capera Espinel | Leidy Andrea Pardo Agudelo | Evelyne Dahanna Torres Sandoval | Zaily del Pilar García Gutiérrez |

Señalado esto, se debe tener en cuenta los recursos humanos con los que cuenta el programa Educa a tu hijo. La selección del personal encargado de velar por el cumplimiento adecuado de aquellas tareas delegadas se divide en promotores y ejecutores. Los promotores juegan un papel fundamental ya que asumen la responsabilidad de capacitar a los ejecutores para que se encarguen de interactuar directamente con las familias hacia su preparación, respetando sus conocimientos, experiencias y saberes; se hace un reconocimiento a la diversidad y por ello se fomenta una educación intercultural.

Como parte de la estrategia de implementación, se desarrollan dos modalidades de atención de acuerdo a la variedad de contextos donde se implemente el programa Educa a tu hijo. La primera modalidad es denominada Atención individualizada, allí la atención se inicia antes, durante y después del embarazo a través de un acompañamiento a los padres de familia efectuado por enfermeras y médicos que tienen como propósito informar ciertos aspectos de cuidado y control en esta etapa; posteriormente, después del nacimiento hasta los dos años de edad, se realiza visitas semanales en las que se explica y se da a conocer a madres y padres de familia los folletos que corresponden al programa. Asimismo, se hace una demostración de las actividades para una mejor comprensión de éstas - que dan paso al desarrollo integral de sus hijos - por parte de los padres de familia (Ferreiro et al, 2002).

La segunda modalidad se denomina Atención grupal y abarca entre los dos y seis años de edad. En esta modalidad se lleva a cabo una "actividad conjunta», puesto que se inicia la participación de familias, niños y niñas junto con el personal orientador - ejecutores_, quienes pretenden alcanzar la participación de todos en pro del desarrollo integral de la infancia. Gracias a dichas actividades se promueve la interacción e intercambio de experiencias, fortaleciendo las relaciones interpersonales de los niños y las niñas con sus padres y la comunidad a la que pertenecen, es allí donde se trabaja en equipo, en comunidad, en sociedad; se comunican, se respetan y se reconocen como personas diversas dentro de un contexto de educación intercultural.

El programa propone nueve folletos como soporte pedagógico. Cada folleto presenta recomendaciones y logros que deben alcanzar los niños y las niñas mediante actividades y acciones diseñadas de acuerdo a su edad —lo que posibilita que se pueda valorar el nivel alcanzado-. Las familias son las encargadas de desarrollar tales actividades para estimular el desarrollo infantil en el hogar.

\section{Programa Hogares Comunitarios de Bienestar - $\mathrm{HCB}-$}

Para proceder con el análisis y contraste con el programa de Cuba Educa a tu hijo, expuesto anteriormente, se realizó la selección del programa del Instituto 
Colombiano de Bienestar Familiar — ICBF-: Hogares Comunitarios de Bienestar — $\mathrm{HCB}$ - el cual brinda apoyo y atención a la primera infancia.

El Instituto Colombiano de Bienestar Familiar a lo largo del tiempo se ha preocupado y ha dirigido la atención a la primera infancia desde una propuesta pedagógica que influye en la vida cotidiana de los niños y niñas, el papel protagónico de la familia y de la comunidad en su desarrollo.

En 1972 se crearon cien Centros Comunitarios para la Infancia — ccl-con el fin de satisfacer las necesidades y prestar atención a los niños y niñas menores de siete años, estos centros ofrecían servicios educativos, preventivos, con participación de la comunidad y las familias. Así mismo, en la Ley 27 del 20 de diciembre de 1974 el ICBF creó los Centros de Atención Integral al Preescolar —CAIP—, en la actualidad conocidos como hogares infantiles; además, gracias al apoyo de la Unicef, se construyó el proyecto Unidad de Estudio y Análisis de Nuevas Modalidades de Atención al Niño para organizar y estructurar las experiencias o necesidades que se evidenciaban en el país, y que estaban relacionadas con el cuidado y educación a niños y niñas pequeños.

La Dirección general del Instituto expidió la Resolución nº 1822 de 1979, a través de la cual señala que los padres de familia y la comunidad debían asumir la administración de los Hogares Infantiles, lo que les reconoció su papel educativo, ya que fueron ellos quienes crearon los proyectos de atención a la primera infancia en los departamentos de Santander y Cauca; de allí, debido al resultado de estos programas, se promovió en el resto del país la creación de modalidades no convencionales.

Estas modalidades y sus jornadas de atención se adecuaron a las necesidades de los niños y niñas, a los hábitos y costumbres y a la disponibilidad de alimentos de cada región. Ello refleja un factor de reconocimiento a la diversidad dentro del contexto colombiano, pues el ICBF con sus programas sólo alcanzaba a cubrir el 7 \% de la población infantil en condición de abandono y desnutrición, por lo que propone la creación de otras estrategias que permitan ampliar la cobertura.

El Consejo Nacional de Política Económica y Social —Conpes_ aprobó a finales de 1986 el proyecto Hogares Comunitarios de Bienestar — HCB - como una estrategia de desarrollo humano con una nueva filosofía de atención para cubrir la población infantil más pobre de zonas urbanas y núcleos rurales.

La atención del programa Hogares Comunitarios de Bienestar — HCB — se presta a través de diferentes actividades bajo el propósito de integrar la acción conjunta del proceso a la familia, la comunidad y el Estado, y, de esa forma, dar apoyo mediante la atención de necesidades básicas de protección, cuidado, nutrición, salud, 
Proceso de educación intercultural en primera infancia: programa Educa a tu hijo -Cuba- y programa Hogares Comunitarios de Bienestar -HCB -Colombia| Cindy Tatiana Capera Espinel | Leidy Andrea Pardo Agudelo | Evelyne Dahanna Torres Sandoval | Zaily del Pilar García Gutiérrez |

educación y desarrollo psicosocial de los niños y niñas en la primera infancia; así, se les garantiza a ellos un desarrollo armónico e integral.

Este programa, según las necesidades de la población, las condiciones del territorio, las fuentes de financiación y las transformaciones propias, ha desarrollado y generado una serie de estrategias o modalidades que son los servicios HCBFAMI —Familia Mujer e Infancia—, нСB Familiares, НСB Agrupados, НСB Múltiples, HCB Empresariales y jardines sociales, los cuales buscan el cumplimiento de los derechos de los niños y niñas en la primera infancia, y el acompañamiento y apoyo a las familias para formar pautas de cuidado, crianza y fortalecimiento de vínculos familiares; igualmente, pretenden estimular en la comunidad la participación en la solución de conflictos, todo eso en pro del bienestar de los niños y niñas en la primera infancia (ICBF, 2014).

A continuación se da a conocer brevemente las características, aspectos particulares, antecedentes, metodología, recursos, entre otros rasgos, de las seis modalidades que se manejan dentro del programa (ICBF, 2014).

Primero se encuentra la modalidad de Familia Mujer e Infancia — FAMI-, la cual va dirigida a mujeres gestantes, madres lactantes, niños y niñas menores de dos años en situación de vulnerabilidad social. El servicio corresponde a la modalidad familiar y opera en la casa del agente educativo comunitario —padre o madre comunitaria-. Cabe resaltar que se prioriza a la comunidad de niveles uno y dos del Sisbén y a familias en condición de desplazamiento o pertenecientes a grupos étnicos.

La segunda modalidad se denomina Hogares Comunitarios de Bienestar — НCB Tradicionales-, este servicio corresponde a la modalidad comunitaria y cuenta con diferentes formas de atención y servicios específicos; allí se encuentran los Hogares Comunitarios de Bienestar Familiar. Este servicio se presta en las viviendas de las personas previamente capacitadas, quienes se responsabilizan del cuidado y atención de un grupo conformado de doce a catorce niños y niñas. Asimismo, se puede atender máximo dos menores que se encuentren en el rango de edad entre los seis y los dieciocho meses y un niño en situación de discapacidad.

En su tercera modalidad, existen los Hogares Comunitarios de Bienestar Agrupados y Múltiples, los cuales son una forma de atención en la que se integran y se agrupan dos o más hogares comunitarios familiares en un mismo centro o planta física. El número de hogares que se puede agrupar depende de la capacidad que tenga la infraestructura.

La cuarta modalidad consiste en los Hogares Comunitarios Empresariales. Este servicio va dirigido a niños y niñas desde los seis meses hasta los cinco años de edad, 
hijos de los trabajadores de más bajos ingresos con el apoyo y cofinanciación de las empresas donde estos laboran.

Por último, los jardines sociales hacen parte de esta modalidad, pues son una forma de atención para niños y niñas que tengan desde seis meses hasta menores de cinco años, los cuales agrupan hasta treintaidós Hogares Comunitarios Familiares. Estos funcionan en un sitio especialmente construido para este fin y son administrados y cofinanciados por Cajas de Compensación Familiar, Fundaciones, ONG y Organismos de Cooperación.

Dentro de la metodología y estrategias de los Hogares Comunitarios de Bienestar - $\mathrm{HCB}$ - el ICBF busca garantizar la práctica de los derechos fundamentales de los niños y las niñas menores de seis años en aras de favorecer su desarrollo y potenciar su calidad de vida bajo los ideales de equidad e inclusión social, pues para tal fin es condición indispensable el respeto a las diferencias humanas y, en consecuencia, a la diversidad étnica y cultural del país (ICBF, 2011).

Es así como surge la implementación de estrategias para el cumplimiento y desarrollo de los derechos fundamentales de los niños y niñas. Entre ellas se encuentra la «Fiesta de la lectura», una estrategia de cualificación orientada a promover acciones para garantizar el derecho de los niños y las niñas a formarse como ciudadanos plenos en el desarrollo del lenguaje, el juego, el arte, los símbolos de la cultura $y$, en general, el desarrollo de lenguajes expresivos. También se encuentra la estrategia «Colombia crece en el cumplimiento de los deberes humanos desde la Primera Infancia», la cual, en cuanto proyecto de formación ciudadana, pretende cambiar los hábitos culturales de violencia para propiciar la constitución de nuevos seres humanos comprometidos con el respeto, el cuidado de sí mismos, de los otros y de la naturaleza. Igualmente, las «Huertas caseras» es una estrategia para promover la seguridad alimentaria, orientada al cumplimiento del derecho a la alimentación y a la salud de los niños y niñas a través de la participación comunitaria y la educación de los padres de familia. Por último, se encuentran los «Comportamientos prosociales», estrategia que tiene el objetivo de promover y fortalecer el desarrollo de las capacidades de las familias, las madres comunitarias, las maestras y otros agentes educativos, para que incidan en la promoción de la prosocialidad y la prevención, en Hogares Comunitarios de Bienestar, de la agresión en niños y niñas de tres a seis años.

Estas estrategias aportan beneficios a los niños y niñas, dado que con su ayuda se garantiza un desarrollo integral y el individuo adopta comportamientos que favorecen su relación con los demás y su identificación en un contexto intercultural.

En síntesis, todo lo anterior permite reconocer y dar valor a los derechos de los seres humanos desde su nacimiento, asegurando las condiciones afectivas, sociales 
Proceso de educación intercultural en primera infancia: programa Educa a tu hijo -Cuba- y programa Hogares Comunitarios de Bienestar -HCB -Colombia| Cindy Tatiana Capera Espinel | Leidy Andrea Pardo Agudelo | Evelyne Dahanna Torres Sandoval | Zaily del Pilar García Gutiérrez |

y materiales suficientes para su desarrollo. En este orden de ideas, se entienden a los niños y niñas de la primera infancia como sujetos de derecho, es decir, sociales, culturales, singulares y activos, capaces de construir sus ideas para convivir en una sociedad participativa. Gracias a dichas actividades se impulsa la interacción e intercambio de ideas y de diferentes experiencias, no sólo de los niños y niñas, sino de las madres comunitarias, padres de familia, la comunidad y el entorno que los rodea. Lo que busca, entonces, es fortalecer las relaciones interpersonales entre ellos por medio del trabajo en equipo y el respeto a cada personalidad dentro de un contexto intercultural.

Es por esto que el país se preocupa por crear proyectos dirigidos a la niñez, la familia y la comunidad en los que se brinde atención y servicios adecuados para el óptimo y pleno desarrollo de sus capacidades y potencialidades.

\section{Una perspectiva intercultural: análisis de programas para la primera infancia en Cuba y Colombia}

Con base en las características e información más relevante de los programas anteriormente nombrados —Educa a tu hijo, correspondiente a Cuba, y Hogares Comunitarios de Bienestar, correspondiente a Colombia-, se procede a dar inicio al análisis comparativo en el que se confrontarán los dos programas para determinar diferencias y posibles semejanzas bajo la perspectiva de la educación intercultural.

En cuanto a semejanzas, Educa a tu hijo y Hogares Comunitarios de Bienestar son programas no convencionales aprobados por los ministerios de educación correspondientes, es decir, programas que no se ejecutan en instituciones educativas formales, ya que su ejecución se realiza fuera de aulas escolares, zonas rurales y algunas urbanas, en definitiva, lugares donde la primera infancia no tiene fácil acceso a escuelas $u$ organizaciones educativas.

El objetivo de cada programa pretende brindar atención integral a la primera infancia y, a su vez, propiciar el desarrollo integral que permita a niños y niñas iniciar y fortalecer su formación personal, cognitiva, corporal, afectiva y social; formación que les permitirá desenvolverse, explorar, adaptarse y distinguir un determinado contexto cultural donde serán reconocidos como sujetos diversos y únicos.

Es posible afirmar, entonces, que los dos programas fomentan una educación intercultural desde y para la primera infancia debido a que su población objeto proviene de diferentes contextos, costumbres, con características propias, siendo esto el reflejo de una diversidad cultural que debe ser reconocida para Ilevar a cabo un proceso de interacción social e intercambio de experiencias. 
Es notorio que cada programa trabaja con una población específica y en pro del desarrollo de la primera infancia. Por ello se desarrollan una serie de modalidades según la metodología aplicada. Sin embargo, dentro del programa Educa a tu hijo sólo se desarrollan dos modalidades y en el programa Hogares Comunitarios de Bienestar se desarrollan seis.

Dentro de las modalidades y propuestas de trabajo de cada programa los niños y niñas, la familia y la comunidad son partícipes y se les reconoce como agentes y protagonistas que contribuyen e intervienen en la formación y desarrollo integral de la primera infancia.

No obstante, dentro del programa Educa a tu hijo se evidencia más participación por parte de la familia y la comunidad, pues son quienes, a partir de la capacitación y el estudio de los folletos que contienen las estrategias, guían, acompañan y lideran de forma constante el proceso de atención integral para la primera infancia. Por el contrario, en el programa Hogares Comunitarios de Bienestar las madres comunitarias asisten a capacitaciones realizadas por el ICBF que les permite ser las encargadas de guiar y llevar a cabo las estrategias que propician la atención integral a la primera infancia, por tal motivo ellas se consideran como las agentes educadoras principales.

En vista de las diferencias y semejanzas que se hallaron en los programas Educa a tu hijo - Cuba- - y Hogares Comunitarios de Bienestar —Colombia-, se retomarán los temas tratados en el artículo a partir del marco de la experiencia en Cuba desde la mirada intercultural y de la formación en pedagogía infantil de la Corporación Universitaria Minuto de Dios - UNIMINUTO, como base que permite esclarecer la importancia y el fundamento de un proceso de educación intercultural para la primera infancia.

La recopilación de todas las características y aspectos de cada uno de los programas se considera el fundamento que da valor y peso al tema central que gira en torno a una educación intercultural, existente en la primera infancia, pero que, en algunas ocasiones, no se reconoce o no se fomenta en el ámbito de formación educativo.

Cabe resaltar que a pesar de que Cuba y Colombia son países diferentes en cuanto a su estructura organizativa, educacional, política, cultural y social, se pudo rescatar ideas, aportes claves y significativos, no sólo para la formación profesional como docentes, sino también humana. Es allí donde, como docentes en formación, podemos tomar conciencia de que se debe asumir el reto de reconocer, aceptar, diseñar y ejecutar programas como los abordados anteriormente y, de igual modo, espacios o ambientes de aprendizaje que den paso a un proceso intercultural que le apueste a un reconocimiento de la diversidad, progreso e inclusión social en un determinado contexto. 
Proceso de educación intercultural en primera infancia: programa Educa a tu hijo -Cuba- y programa Hogares Comunitarios de Bienestar -HCB -Colombia| Cindy Tatiana Capera Espinel | Leidy Andrea Pardo Agudelo | Evelyne Dahanna Torres Sandoval | Zaily del Pilar García Gutiérrez |

\section{Conclusiones}

Buscar y comparar los programas educativos dirigidos a la primera infancia en países como Cuba y Colombia posibilita responder ciertos interrogantes que surgieron desde la experiencia obtenida en el programa de corta duración en Cuba. Efectivamente, en este lugar se presentó la oportunidad de contrastar teoría y práctica, en relación con la educación implementada, durante una visita a un circulo infantil - centro de estimulación y desarrollo para niños con necesidades especiales_, en la que se evidenció un proceso de integración intercultural en el contexto educativo - tema central del presente artículo-; se pretendió, pues, dar solución a preguntas como: ¿existe alguna entidad o programa dirigido a la primera infancia que vele por la educación y por un proceso de desarrollo integral? ¿A qué necesidades de la primera infancia se da respuesta en un país como Cuba? ¿Tiene relación la metodología implementada en Cuba con la metodología implementada en Colombia?

Lo anterior permite determinar y dirigir la atención a una problemática específica: la educación intercultural como proceso de desarrollo integral para niños y niñas de zonas rurales que por su lejana ubicación no pueden acceder a una educación formal. Es allí donde nacen los programas que buscan satisfacer esta necesidad, siendo el programa Educa a tu hijo - Cuba- y los Hogares Comunitarios del ICBF - Colombia - una posible respuesta y solución. Respecto a estos programas de educación no formal, dirigidos a una misma población, es válido resaltar la importancia y la metodología de cada uno a la hora del trabajo con la primera infancia y la implementación de una educación intercultural, en la que niños, niñas, familias y comunidad realizan un cruce de experiencias, saberes, aprendizajes y costumbres que van construyendo una educación intercultural dentro de un contexto social donde se reconoce la diversidad como alternativa de aprendizaje y de valor cultural.

Es debido a esto que se puede concluir, a partir del perfil profesional como licenciadas, la relevancia de tener experiencias que posibiliten conocer, interactuar e identificar contextos educativos, sociales y culturales que permitan cuestionar la práctica docente y el sistema educativo, para que, con base en ello, como docentes se logre adquirir habilidades, conocimientos y capacidades que se conviertan en las herramientas para dar respuesta a interrogantes y necesidades por medio de la investigación, la cual es la esencia de la formación e innovación.

Finalmente, indagar y conocer la estructura de dichos programas, que sirven como guía para la construcción de ambientes de aprendizaje con un enfoque intercultural, permite ampliar la visión en cuanto a la metodología y recursos que podemos implementar en la práctica docente, lo cual busca forjar una práctica educativa innovadora en la que la interculturalidad sea el eje central en el desarrollo integral de niños y niñas. 


\section{Referencias}

Agudelo Cely, N., y Estupiñán Quiñones, N. (2009). La sensibilidad intercultural en Paulo Freire. Revista Historia de la Educación Latinoamericana, 13, 85-100. Recuperado de http://www.redalyc.org/articulo.oa?id=86912384006>

Aranda Viollegas, G., Roman Lorente, C., y Gómez López, M. (s.f.). La multiculturalidad como objeto de atención a la diversidad. Recuperado de de: http://rabida.uhu.es/dspace/ bitstream/handle/10272/6619/Multiculturalidad_diversidad.pdf?sequence=2

Baronnet, B. (2013). La autonomía como condición para la educación intercultural. México.

Cespedes, N. (2010). La educación popular en la lucha por el buen vivir, educación popular, interculturalidad y buen vivir, la paz/ el salvador 2010. Recuperado de http://www.ceaal. org/v2/archivos/randina/Separata\%20CEAAL.pdf

Congreso de la República de Colombia. (2006, 08 de noviembre). Ley 1098: por la cual se expide el código de la infancia y la adolescencia. Diario oficial, 1-48. Recuperado de http://www.oei.es/quipu/colombia/codigo_infancia.pdf

Díaz Cano, L., Pulido Álvarez, A., y Giraldo Huertas, J. (2011). Una mirada a la caracterización de la primera infancia. Contextos y métodos, 13(1), 279-293. Recuperado de http://www. scielo.org.co/scielo.php?script=sci_arttext\&pid=S1692-715X2015000100017

Directrices de la Unesco sobre la educación intercultural. (s.f.). Recuperado de http://unesdoc. unesco.org/images/0014/001478/147878s.pdf

Dra. Peralta, M. (2014). Organización de Estados Iberoamericanos para la Educación, la Ciencia y la Cultura (OEI). Arte, Educación y Primera Infancia: sentido y experiencia (Interculturalidad, primera Infancia y artística). Recuperado de http://www.oei.es/ publicaciones/LibroMetasInfantil.pdf

Escarbajal, A. (2014). La educación intercultural en los centros educativos. Electrónica Interuniversitaria de Formación del Profesorado, 17(2), 29-43. Recuperado de http://www. redalyc.org/articulo.oa?id=217031054004

Escobar, F. (2006). Importancia de la educación inicial a partir de la mediación de los procesos cognitivos para el desarrollo humano integral. Laurus, Revista de educación, 12(21), 169194. Recuperado de http://www.redalyc.org/pdf/761/76102112.pdf

Fandiño, G. (2012). Una propuesta pedagógica para la educación de la primera infancia. Ministerio de educación Nacional. Recuperado de http://www.colombiaaprende.edu.co/ html/familia/1597/articles-310603_docu2.pdf

Ferreiro Rivera, I., Siverio Gómez, A., Domínguez Pino, M., Yañez Sterling, H., Pérez Moran, M., y Burke Beltrán, M. (2002). Educa a tu hijo. La experiencia cubana en la atención integral al desarrollo infantil en edades temprana. Cuba: Unicef/ CELEP/ MINED.

González, E. (2005). Estado, nación y relaciones interculturales en Hispanoamérica: de la colonia a la República. Una interpretación cubana de la cuestión mapuche. Cuba: Facultad de filosofía e historia. 
González, I. (2011). Educación intercultural en aulas de educación infantil. Recuperado de http://www.auladelpedagogo.com/2011/02/educacion-intercultural-en-aulas-deeducacion-infantil/.

ICBF —Instituto Colombiano de Bienestar Familiar-. (2014). Macroproceso gestión para la atención integral a la primera infancia, "lineamiento técnico, administrativo y operativo, modalidad Hogares Comunitarios de Bienestar en todas sus formas -Fami, familiares, grupales, múltiples, múltiples empresariales y jardines sociales— para la atención a niños y niñas hasta los cinco (5) años de edad». Recuperado de http://www.normoteca.co/ normahtml/000003610100690000000301.pdf

ICBF —Instituto Colombiano de Bienestar Familiar-. (2011). Guía para la implementación del proyecto pedagógico educativo comunitario en la primera infancia. Recuperado de http://www.icbf.gov.co/portal/page/portal/PortallCBF/macroprocesos/misionales/primerainfancia/5/GUIA\%20PARA\%20LA\%20IMPLEMENTACI\%C3\%93N\%20PPEC.pdf

Lazos Chavero, E. (2013). Interculturalidad: naturalezas dominadas, naturalezas vividas. México.

MEN —Ministerio de Educación Nacional-. (2009). Desarrollo Infantil y Competencias en la Primera Infancia. Colombia. Recuperado de http://www.mineducacion.gov.co/ primerainfancia/1739/articles-282097_archvo_pdf_documento10.pdf

MEN - Ministerio de Educación Nacional-. (2013). ¿Qué es la educación inicial? Bogotá, Colombia. Recuperado de http://www.mineducacion.gov.co/primerainfancia/1739/w3article-316845.html

MEN —Ministerio de Educación Nacional. (2013). Modalidades de educación inicial. Bogotá, Colombia. Recuperado de http://www.mineducacion.gov.co/primerainfancia/1739/w3article-228881.html

Meszaros. I. (2007)._Educación más allá del capital, Revista Educación superior cifras y hechos, 5(31-32), 23-58. Recuperado de http://computo.ceiich.unam.mx/webceiich/ docs/revis/33Bol031.pdf

Muñoz Barriga, A. (2013). Diversidad cultural en la primera infancia: un reto educativo en contextos urbanos. Educación y ciudad, (24), 119-131. Recuperado de http://www.idep. edu.co/revistas/index.php/educacion-y-ciudad/article/view/71/59

Sáez Alonso, R. (2006). La educación intercultural. Revista de Educación, Universidad Complutense de Madrid, 859-881. Recuperado de http:/www.revistaeducacion.mec.es/ re339/re339a37.pdf

Siverio Gómez, A. (2011). La contextualización del modelo de atención educativa no institucional cubano "Educa a tu hijo» en países latinoamericanos. Unicef. Recuperado de http://www.movilizando.org/images/Educa_a_tu_Hijo_UNICEF_Siverio.pdf

Umayahara, Mami. (2004). En búsqueda de la equidad y calidad de la educación de la primera infancia en América Latina. Revista Latinoamericana de Ciencias Sociales, Niñez y Juventud, 2(2), 21-49.

Unicef — Fondo de las Naciones Unidas para la Primera Infancia-. (2011). La contextualización del modelo de atención educativa no institucional cubano "Educa a tu hijo" en países latinoamericanos. Recuperado de file:///C:/Users/USER/Downloads/Educa_a_tu_Hijo_ UNICEF_Siverio.pdf 
Walsh, C. (2009). Interculturalidad crítica y educación intercultural. La Paz, Bolivia. Recuperado de: http://www.saludpublica.uchile.cl/u/download.jsp?document=110597\&property=atta chment\&index=0\&content $=$ null

Walsh, C. (s.f.). Interculturalidad crítica y pedagogía de-colonial: apuestas (des)de el insurgir, re-existir y re-vivir. Recuperado de https://redinterculturalidad.files.wordpress. com/2014/02/interculturalidad-crc3adtica-y-pedagogc3ada-decolonial-walsh.pdf

Cómo citar: Capera, C., Pardo, L., Torres, E. y García, Z. (2017).

Proceso de educación intercultural en primera infancia: programa Educa a tu hijo, Cuba y programa Hogares Comunitarios de Bienestar, HCB, Colombia. Praxis Pedagógica, 21 (119-142). 